\title{
A Linear Time Heuristics for Trapezoidation of GIS Polygons
}

\author{
Gian Paolo Lorenzetto and Amitava Datta \\ Department of Computer Science \& Software Engineering \\ The University of Western Australia \\ Perth, W.A. 6009 \\ Australia email : \{gian,datta\}@cs.uwa.edu.au
}

\begin{abstract}
The decomposition of planar polygons into triangles is a well studied area of Computer Graphics with particular relevance to GIS. Trapezoidation is often performed as a first step to triangulation. Though a linear time algorithm [2] for the decomposition of a simple polygon into triangles exists, it is extremely complicated and in practice $O(n \log n)$ algorithms are used. We present a very simple $O(n)$-time heuristics for the trapezoidation of simple polygons without holes. Such polygons commonly occur in Geographic Information Systems (GIS) databases.
\end{abstract}

\section{Introduction}

The decomposition of a simple polygon into triangles is a well studied field of computational geometry and computer graphics [1]. In computer graphics and GIS applications, it is much easier to render a simple shape like a triangle compared to a large polygon. Similarly in computational geometry, a large number of data structures related to polygons are based on triangulations of these polygons. For many triangulation algorithms, a trapezoidal decomposition of the polygon is computed as a first step for triangulation. A trapezoid is a four-sided polygon with a pair of opposite sides parallel to each other. The main difference between a trapezoidal decomposition and a triangulation is that the vertices of the trapezoids can be additional points on the boundary of the polygon, whereas, in a triangulation it is not allowed to introduce new vertices.

The best known triangulation algorithm is by Chazelle [2] and it runs in $O(n)$ time, where $n$ is the number of vertices of the polygon. However, Chazelle's algorithm is extremely complicated and difficult to implement. Earlier, Garey et al. [5] designed an $O(n \log n)$ algorithm for triangulation by dividing the input polygon into monotone pieces. Even now, the algorithm by Garey et al. [5] is extensively used for its simplicity and programming ease. Later, Chazelle and Incerpi [3] improved upon this algorithm by introducing the notion of sinuosity which is the number of spiraling and anti-spiraling polygonal chains on the polygon boundary. The algorithm by Chazelle and Incerpi [3] runs in $O(n \log s)$ time, where $s$ is the sinuosity of the polygon. The sinuosity of a polygon is usually a small constant in most practical cases and the algorithm in [3] is an 
almost linear-time algorithm. However, the algorithm by Chazelle and Incerpi [3] is based on a divide-and-conquer strategy and still quite complex.

In this paper, we are interested in the trapezoidal decomposition of a simple polygon rather than a triangulation. In computational geometry, a triangulation in performed for building efficient data structures based on this triangulation. Such data structures are used for many applications e.g., for processing shortest path queries or ray shooting. On the other hand, in almost all applications in computer graphics and geographic information systems (GIS), the main requirement is the fast rendering of a polygon. A trapezoid is as simple a shape as a triangle and a trapezoid is easy to render like a triangle in raster graphics devices. In this paper, we present an $O(n)$-time simple heuristics for computing trapezoidal decomposition of a simple polygon without holes. Our heuristics can be used for fast rendering of large GIS data sets which quite often consist of polygons with thousands or hundreds of thousands of vertices.

The fastest known algorithm for trapezoidation of general GIS data sets is by Lorenzetto et al. [6]. Their algorithm improve upon a previously published algorithm by Žalik and Clapworthy [7] and can do trapezoidal decomposition of general GIS polygons with holes in $O(n \log n)$ time, where $n$ is the total number of vertices for the polygon and all holes inside it. However, many GIS polygons do not contain holes inside them and it is interesting to investigate whether it is possible to design a simpler and faster algorithm for this problem when the polygons do not contain holes. Our algorithm is simple and terminates in $O(n)$ time for most polygons encountered in GIS applications, where $n$ is the number of vertices in the polygon. We call the algorithm as a linear-time heuristics since we are unable to prove a tight theoretical upper bound for the running time of the algorithm. However for almost all polygons encountered in GIS data sets, the running time is $O(n)$ with the hidden constant in big-Oh very small. However, our algorithm always produces a correct trapezoidation and this is ensured through an in-built correctness test in the algorithm.

The rest of the paper is organized as follows. We discuss some preliminaries and an algorithm for trapezoidal decomposition of monotone polygons in Section 2. We present our $O(n)$ time heuristics in Section 3 and finally we discuss the complexity of the heuristics and our experimental results in Section 4.

\section{Terminology}

We consider only simple polygons without holes inside them and the edges of the polygons intersect only at end points. A trapezoid is a four-sided polygon with a pair of opposite sides parallel to each other. In this paper, all trapezoids have two sides parallel to the $x$-axis. We assume that no two vertices are on the same line parallel to the $x$ or the $y$ axis. In other words, no two vertices have the same $y$ or $x$ coordinates. This assumption simplifies our presentation considerably but we do not impose this restriction in our implementation. The algorithm processes vertices based on their type. We consider three types of vertices depending on the two end points of the two edges incident on the vertex. In the following, we 
use the notion of the left and right side of the plane for a directed edge $\overline{u v}$ of the polygon. If the edge is directed from $u$ to $v$, we assume that an observer is standing at $u$, facing $v$ and the notion of the left or right side is with respect to this observer.

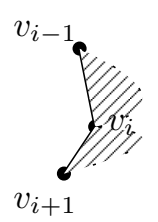

INT

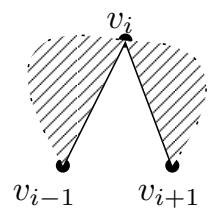

MAX

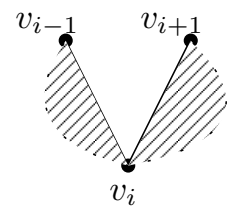

MIN

(a)

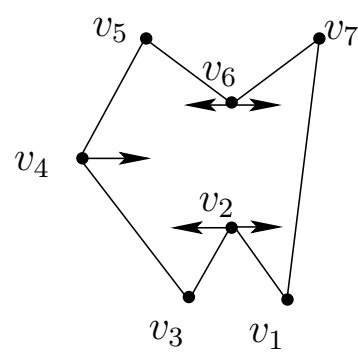

(b)

Fig. 1. (a) The three vertex types used in the algorithm. (b) The assignment of sweep lines. $v_{4}$ is of type INT, $v_{2}$ is of type MAX and $v_{6}$ is of type MIN. The other four vertices do not throw sweep lines.

Each vertex $v_{i}$ is classified as one of the following types.

- INT if the neighboring vertices of $v_{i}$ have a greater and a lower $y$-coordinate, respectively, than $v_{i}$. The interior of the polygon is to the left sides of the directed edges $\overline{v_{i-1} v_{i}}$ and $\overline{v_{i} v_{i+1}}$.

- MIN if both neighboring vertices have a greater $y$-coordinate than $v_{i}$. The interior of the polygon is to the right sides of the directed edges $\overline{v_{i-1} v_{i}}$ and $\overline{v_{i} v_{i+1}}$.

- MAX if both neighboring vertices have a lower $y$-coordinate than $v_{i}$. The interior of the polygon is to the left sides of the directed edges $\overline{v_{i-1} v_{i}}$ and $\overline{v_{i} v_{i+1}}$.

Note that all the vertices can be classified according to these three categories by traversing the polygon boundary once. In our algorithm, a vertex of type INT may become a corner vertex of a trapezoid. We indicate this by assigning a sweepline to an INT vertex. This sweepline starts at the INT vertex and goes either towards left or right depending on which side the interior of the polygon is. Similarly, a vertex of type MAX supports the bottom edge of a trapezoid and sweeplines may be thrown in both directions to determine the edge. A MIN vertex supports a top edge of a trapezoid and hence sweeplines are thrown in both directions. The different vertex types are shown in Figure 1(a). The shaded parts in Figure 1(a) denote the interior of the polygon. An example polygon with directions for sweeplines for its vertices is shown in Figure 1(b). Note that the directions of the sweeplines can be stored in the same pass of the polygon 
boundary while determining the vertex types. This computation takes $O(n)$ time when $n$ is the number of vertices.

\subsection{A trapezoidation algorithm for monotone polygons}

A monotone polygon is a polygon such that the polygon boundary can be decomposed into two chains by splitting the polygon at the two vertices with maximum and minimum $y$ coordinates. These two chains are monotone with respect to the $y$-axis. In other words, a monotone polygon can have only vertices of type INT and no MAX or MIN vertices. Note that the vertex with highest (resp. least) y coordinate is not a MAX (resp. MIN) vertex according to our definition. Let $v_{j}$ be the vertex with highest $y$-coordinate and $v_{j-1}$ and $v_{j+1}$ are the vertices just before and after $v_{j}$ during a clockwise traversal of the polygonal boundary. Then the interior of the polygon is towards the right of the two directed edges $\overline{v_{j-1} v_{j}}$ and $\overline{v_{j} v_{j+1}}$. This violates the definition of a MAX vertex. It is easy to show in a similar way that the vertex with minimum $y$-coordinate is not a MIN vertex.

Our algorithm is based on a traversal of the polygon boundary and throwing sweeplines from each vertex. We first determine the direction in which each vertex throws sweepline in a preprocessing step as explained before. Our main task is to determine where each of the sweeplines intersect the polygon boundary. To determine this, we traverse the polygon boundary twice starting from $v_{\text {min }}$, the vertex with the minimum $y$-coordinate. The traversals are first in the clockwise and then in the counter-clockwise direction. We use a stack for holding the vertices which we encounter. In the following, by $v_{t o p}$ we mean the vertex at the top of the stack. The stack is empty initially. During the clockwise traversal of the polygon boundary, we push a vertex $v_{k}$ on the stack if $v_{k}$ throws a sweepline towards right. When we encounter vertex $v_{i}$, we execute the following steps.

- We check whether a horizontal sweepline towards right from $v_{t o p}$ intersects the edge $\overline{v_{i} v_{i+1}}$. If there is an intersection, we mark the intersecting point as a vertex of a trapezoid. In this case, $v_{t o p}$ is removed from the stack.

- If $v_{i}$ throws sweepline to the right, we push $v_{i}$ onto the stack.

We execute a similar counter-clockwise traversal for vertices which throw sweeplines towards left. The trapezoids are reported in the following way. We store the intersection of the sweeplines with the edges in a separate data structure. For example, for an edge $e_{i}$, all the intersections of sweeplines with $e_{i}$ are stored as a linked list of intersection points. These intersection points can be kept ordered according to $y$-coordinate easily. We can report the trapezoids starting from $v_{\min }$ and checking these intersection points as we move upwards.

\section{An $O(n)$-time algorithm for general polygons}

The approach of the previous section does not work for more general polygons containing vertices of types MAX and MIN. The main problem is that the sweepline-edge intersections are not always correct. 
In our algorithm, we use three stacks, one each for MAX, MIN, and INT vertex types. The only difference is that when pushing a vertex onto the stack, we check the vertex type and the vertex is pushed onto the correct stack. Similarly, previously an edge was tested against the top of the stack for an intersection, whereas now the edge must be tested against the top of all three stacks.

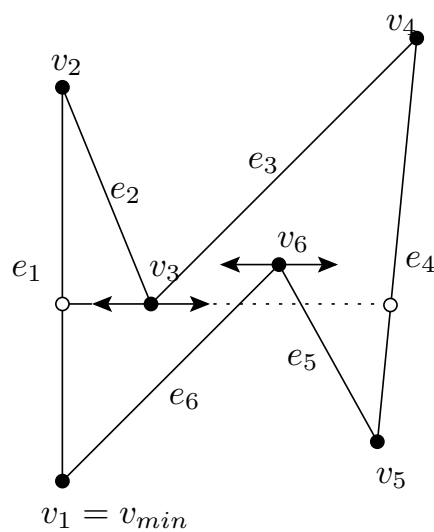

(a)

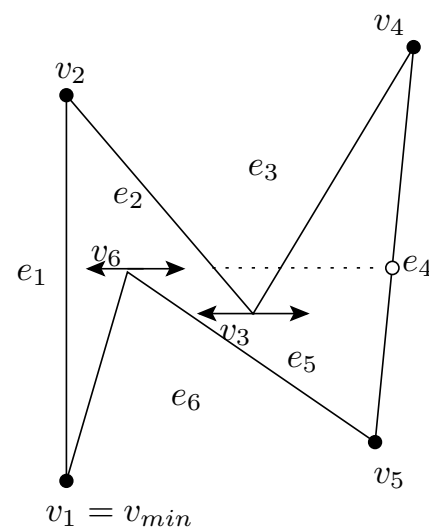

(b)

Fig. 2. Illustrations for incorrect trapezoidation. (a) After a clockwise traversal vertex $v_{3}$ has thrown a sweepline right and caused an incorrect intersection with edge $e_{4}$. (b) After a counter-clockwise traversal vertex $v_{6}$ has thrown a sweepline right and caused an incorrect intersection with $e_{4}$. Both traversals start at $v_{\text {min }}$.

Consider Figure 2(a) and in particular vertex $v_{3}$. During a counter-clockwise traversal $v_{3}$ correctly throws a sweepline to the left intersecting edge $e_{1}$. However it does not throw a sweepline to the right as $v_{3}$ is seen after edges $e_{6}, e_{5}$, and $e_{4}$ - the only edges a sweepline right from $v_{3}$ would intersect.

Note that in a clockwise traversal edge $e_{4}$ is seen before $e_{6}$. For correctness a sweepline right from $v_{3}$ must intersect edge $e_{6}$, but as $e_{4}$ is seen first, the right intersection from $v_{3}$ is calculated with $e_{4}$, and $v_{3}$ is then popped from the stack. In situations like this, we need to check whether the sweepline is correctly resolved, i,e., it intersects the correct edge. A similar situation is shown in Figure 2(b) when a counter-clockwise traversal causes a wrong intersection for vertex $v_{6}$. We denote the $x$ and $y$ coordinates of a point $p_{i}$ by $x_{i}$ and $y_{i}$. Consider a MIN vertex $v_{i}$ and a MAX vertex $v_{j}$. Suppose the sweeplines towards right from $v_{i}$ and $v_{j}$ intersect an edge $e_{k}$ at the points $p_{i}$ and $p_{j}$ respectively. Then the following lemma holds.

Lemma 1. If $y_{i}<y_{j}$, then the sweepline towards right (i) either from $v_{i}$ or (ii) from $v_{j}$, cannot be resolved correctly on the polygonal chain from $v_{i}$ to $v_{j}$. 
Proof. First note that the sweepline towards right from the MAX vertex $v_{j}$ has a greater $y$ coordinate compared to the sweepline from the MIN vertex $v_{i}$. Also, $v_{j}$ must have been encountered after $v_{i}$ during a clockwise traversal starting from $v_{\min }$. Otherwise, the sweepline from $v_{j}$ would have intersected either the edge with $v_{i}$ as an endpoint or some other edge before reaching $v_{i}$.

Suppose the sweepline from $v_{i}$ is resolved on an edge $e_{k}$ on the polygonal chain from $v_{i}$ to $v_{j}$. Note that $v_{j}$ has a higher $y$ coordinate compared to $v_{i}$. There are two possibilities. In the first case, the sweepline from $v_{i}$ intersects some edge on the polygonal chain from $v_{j}$ to $v_{\min }$. This is the case in Figure 2 (a). The vertices $v_{3}$ and $v_{6}$ play the role of $v_{i}$ and $v_{j}$ and the edge $e_{4}$ plays the role of $e_{k}$ in this case. The other possibility is that the sweepline from $v_{j}$ (towards right) intersects some edge on the polygonal chain from $v_{\min }$ to $v_{i}$. This is the case shown in Figure 2(b). Hence, either the sweepline from $v_{i}$ or the sweepline from $v_{j}$ cannot be correctly resolved on the polygonal chain from $v_{i}$ to $v_{j}$.

In our algorithm, we produce a correct trapezoidation iteratively and after each iteration we need to check the correctness of the trapezoidation. This check is based on a generalization of Lemma 1. Consider an edge $e_{k}$ with two end points $v_{k}$ and $v_{k+1}$. We call the polygonal chain from $v_{\text {min }}$ to $v_{k}$ (resp. $v_{k+1}$ to $v_{\text {min }}$ ) during a clockwise traversal as the chain before $e_{k}$ (resp. after $e_{k}$ ) and denote by before $\left(e_{k}\right)$ (resp. after $\left.\left(e_{k}\right)\right)$. Suppose in the previous iteration a set of vertices on before $\left(e_{k}\right)$ and after $\left(e_{k}\right)$ had their sweeplines resolved on $e_{k}$. We denote the minimum $y$-coordinate among all the vertices on before $\left(e_{k}\right)$ that are resolved on $e_{k}$ by $b_{k}$ and similarly the maximum $y$-coordinate among all the vertices on after $\left(e_{k}\right)$ that are resolved on $e_{k}$ by $a_{k}$. Then the following lemma allows us to check the correctness of our trapezoidation.

Lemma 2. A trapezoidation is correct if and only if for every edge $e_{k}, 1 \leq k \leq$ $n, b_{k}>a_{k}$.

Proof. First assume that there is at least one edge $e_{k}$ such that $b_{k}<a_{k}$. From Lemma 1 it is clear that the vertex on before $\left(e_{k}\right)$ that is responsible for $b_{k}$ has not resolved its sweepline correctly. Hence, the presence of at least one such edge $e_{k}$ makes the trapezoidation incorrect.

To prove the other direction, assume that for every edge $e_{k}, b_{k}>a_{k}$. In that case, all the sweeplines are resolved correctly and hence the trapezoidation is correct.

In Figure 2, for clockwise traversal, the polygonal chain from $v_{1}$ to $v_{4}$ is before $\left(e_{4}\right)$ and the polygonal chain from $v_{5}$ to $v_{1}$ is after $\left(e_{4}\right) . b_{4}$ and $a_{4}$ are the $y$ coordinates of the intersections on the edge $e_{4}$ by vertices on before $\left(e_{4}\right)$ and after $\left(e_{4}\right)$. In this case, $b_{4}$ is determined by $v_{3}$ and $a_{4}$ is determined by $v_{6}$. The trapezoidation is incorrect since $y_{3}<y_{6}$.

It is clear that if we identify condition of Lemma 1 , we cannot resolve one of the sweeplines, either from $v_{i}$ or from $v_{j}$, on the polygonal chain from $v_{i}$ to $v_{j}$. In the first case, we should try to resolve the sweepline from $v_{i}$ only after 
crossing $v_{j}$ during the clockwise traversal of the polygon. In the second case, we should try to resolve the sweepline from $v_{j}$ only after crossing $v_{i}$ during a counter-clockwise traversal of the polygon. A simple heuristics to overcome the problem described above is to extend the stack approach to stack-of-stacks of vertices, in effect restricting which edges see which sweeplines. In the above example from Figure 2 the problem is caused by the overlapping MAX and MIN vertices. This causes the order in which edges are seen to be incorrect.

\subsection{Preprocessing}

The preprocessing stage classifies each vertex based on type. Once a vertex has been classified it is assigned sweeplines. We assign an initial intersection point for each vertex in the preprocessing stage itself. In general some of these initial intersections are incorrect and they are refined in the next stage of the algorithm. Intersections are calculated for each vertex that throws a sweepline. The first vertex tested is $v_{\text {min }}$. From $v_{\text {min }}$ each vertex is tested in order, in both clockwise and counter-clockwise directions. The polygon boundary is traversed twice for the following reason. For a sweepline-edge intersection to occur the vertex must be seen before the edge. This is necessary because the vertex must be on the stack when the edge is encountered. In some cases this will only occur during a clockwise traversal of the polygon boundary, and in others only during a counter-clockwise traversal. During the pre-processing stage only MAX and MIN vertices are considered, all other vertices are ignored. The following deals with MAX vertices, but the case for MIN is identical. As a sweeping MAX vertex is encountered the edge containing the intersection is updated to note that it has been intersected by a MAX vertex. Once both the clockwise and counterclockwise passes are complete all edges intersected by MAX vertices are marked.

At the end of the preprocessing, each edge potentially stores two pieces of information. An array min of $y$-coordinates for intersections of all the sweeplines thrown by the vertices of type MIN and an array max of $y$-coordinates for intersections of all the sweeplines thrown by vertices of type MAX. These intersections occur according to increasing $y$ coordinates for vertices of type MIN and according to decreasing $y$-coordinates for vertices of type MAX. Also, overall there are at most $2 n$ intersections since each sweepline intersects only one edge. Hence, these two arrays are maintained in linear time during preprocessing.

By $y_{\min }$ we denote the minimum $y$-coordinate in the array $\min$ and by $y_{\max }$ the maximum $y$-coordinate in the array $\max$. This information is used for checking the condition of Lemma 1 . Note that not all intersections are correct at this stage. Consider again Figure 2. After pre-processing vertex $v_{3}$ still throws a sweepline to the right incorrectly, intersecting edge $e_{4}$.

\subsection{Main iteration}

We now discuss the main iterations of our algorithm. Each iteration has two parts, first a trapezoidation step and then a testing stage. We compute a trapezoidation first and then test whether the trapezoidation is correct by checking 
the condition of Lemma 2. We proceed to the next iteration if the trapezoidation is incorrect otherwise the algorithm terminates after reporting the trapezoids.

The trapezoidation in each iteration proceeds similar to the second part of the preprocessing stage, one clockwise and one counter-clockwise traversal of the polygon boundary. As in Section 2.1, we consider vertices of type INT which throw sweepline towards right during the clockwise traversal and vertices of type INT which throw sweeplines towards left during the counter-clockwise traversal. However, we consider vertices of types MAX and MIN during both the traversals. A vertex of type MIN or MAX is pushed onto the corresponding stack when encountered and popped from the stack when both the sweeplines from such a vertex is resolved. The MAX/MIN intersection information stored in the pre-processing stage is used to control which vertices are tested against each edge. A vertex can cause a sweepline-edge intersection left, or right, during both the clockwise and counter-clockwise traversals. As an edge $e_{i}$ is encountered, we take the following actions with respect to $v_{t o p}$, the top vertices in each of the three stacks for MAX, MIN and INT vertices. We denote the $y$-coordinate of $v_{\text {top }}$ by $y_{\text {top }}$. The following steps are taken when $v_{\text {top }}$ is the top vertex in the MIN stack. The actions are similar when $v_{t o p}$ is the top vertex in the other two stacks.

- We check whether a sweepline from $v_{t o p}$ intersects $e_{i}$. If there is an intersection, we check whether the condition in Lemma 1 is violated. For example, if $v_{\text {top }}$ is a MIN vertex, this condition will be violated if $y_{t o p}<y_{\max }$. Suppose, a vertex $v_{j}$ is responsible for the $y_{\max }$ value. We now initialize three new stacks for the three types of vertices. This action can be viewed as maintaining three data structures which are stack-of-stacks, one each for vertex types MIN, MAX and INT. We push three new empty stacks on top of each of the three stack-of-stacks. Note that the sweepline from the vertex $v_{t o p}$ cannot be resolved until we encounter the vertex $v_{j}$, and hence we need to maintain these new stacks until we reach $v_{j}$.

- We delete $y_{t o p}$ from the array min and if necessary, update $y_{\min }$.

- If both (in case of MIN or MAX) or one (in case of INT) sweepline(s) of $v_{t o p}$ is resolved, we pop $v_{t o p}$ out of the stack-of-stacks. In other words, $v_{t o p}$ is popped from the top-most stack of one of the three stack-of-stacks depending on its type.

- If the vertex $v_{i}$ of edge $e_{i}$ throws sweeplines, we push $v_{i}$ into the correct stack-of-stacks depending on its type.

Once both the clockwise and counter-clockwise traversals are over, we test the condition of Lemma 2 for each edge of the polygon. This is done by comparing $y_{\min }$ and $y_{\max }$ for each of the edges. If at least one edge violates the condition, we proceed to the next iteration. Otherwise we report the trapezoids and the algorithm terminates.

Note that the motivation for using a stack-of-stacks data structure is the property discussed in Lemma 1 . The current top stack in a stack-of-stacks takes care of the polygonal chain between a MIN vertex and a corresponding MAX 
vertex when these two vertices are interleaved, i.e., the $y$ coordinate of the MAX vertex is higher than the $y$-coordinate of the MIN vertex as in Figure 2. An old stack is removed from the stack-of stacks when we reach a matching MAX vertex. For example, in Figure 2(a) when we are doing a clockwise traversal, we initialize a new stack when we are at edge $e_{4}$ and find the overlapping of MIN/MAX pair of $v_{3}$ and $v_{6}$. We remove this stack when we reach $v_{6}$ and the sweepline from $v_{3}$ is correctly resolved after that.

When the algorithm terminates, we report the trapezoids by traversing the arrays min and $\max$ for each of the edges in the polygon. These are the correct intersections of sweeplines and vertices and hence the supports of the trapezoids. We omit the details of the reporting of trapezoids in this version. and refer to Figure 3 for an example.

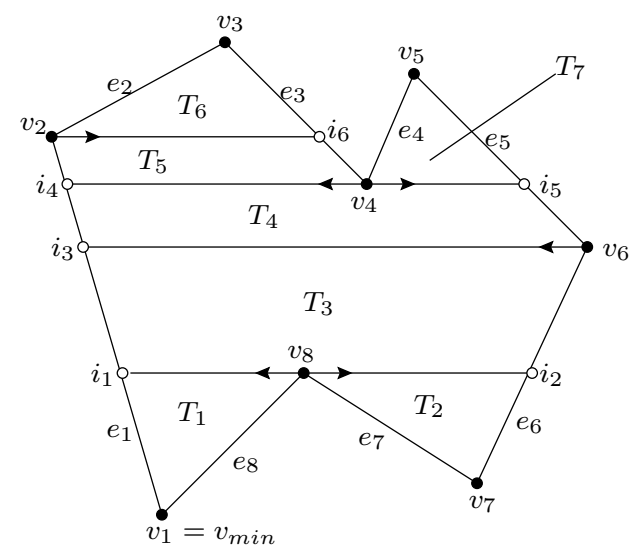

Fig. 3. An example polygon decomposed into trapezoids after the termination of our heuristics.

\section{Experimental Results and conclusion}

The preprocessing stage as well as each iteration of the heuristics takes $O(n)$ time. The reporting of the trapezoids also takes $O(n)$ time. However, we are unable to prove a theoretical upper bound for the heuristics at this point. We strongly suspect that there is a close relationship between the number of iterations required for producing a correct trapezoidation and the sinuosity of the polygon as defined by Chazelle and Incerpi [3]. It is quite difficult to generate polygons with high sinuosity and we have tested our algorithm for polygons up to sinuosity 5 and the number of iterations required are always equal to the sinuosity of the polygon up to this point. Hence, we conjecture that the theoret- 
ical upper bound for the running time of our heuristics is $O(n s)$, where $s$ is the sinuosity of the polygon.

However, most GIS polygons from the GIS Data Depot [4] are polygonal data for land subdivisions, property boundaries and roadways. It is not common to have a high sinuosity for such polygonal data. For all GIS polygons that we have used, the algorithm terminates in four or five iterations. We used a Celeron 400 PC with $328 \mathrm{MB}$ of RAM running Linux Redhat 6 as our experimental platform. We implemented the algorithm by Lorenzetto et al. [6] and the present algorithm, both in $\mathrm{C}++$ using the $\mathrm{g}++$ compiler. Some of the results are shown in Table 1. The present algorithm becomes more efficient for polygons with a large number of vertices and we expect that it will be useful in practice when it is necessary to do trapezoidal decomposition of a large number of polygons very fast.

Table 1. The comparison between our algorithm and the $O(n \log n)$ algorithm by Lorenzetto et al. [6]. All running times are in seconds.

\begin{tabular}{|c|c|c|}
\hline Number of vertices & $O(n \log n)$ algorithm in & \begin{tabular}{c|c|}
{$[6]$} & Our $O(n)$ heuristics \\
\end{tabular} \\
\hline$\overline{5000}$ & 0.12 & 0.11 \\
\hline 10000 & 0.26 & 0.21 \\
\hline 20000 & 0.50 & 0.42 \\
\hline 30000 & 0.76 & 0.64 \\
\hline
\end{tabular}

We plan to investigate the worst-case complexity of our algorithm and construct some worst-case example polygons. This will help us to check whether such worst-case polygons occur in practice in GIS data sets. We also plan to test our algorithm more extensively on GIS data sets in order to optimize our code further.

\section{References}

1. M. Bern, Handbook of Discrete and Computational Geometry, Chapter 22, CRC Press, pp. 413-428, 1997.

2. B. Chazelle, "Triangulating a simple polygon in linear time", Discrete and Computational Geometry, 6, pp. 485-524, 1991.

3. B. Chazelle and J. Incerpi, "Triangulation and shape complexity", ACM Trans. Graphics, 3, pp. 153-174, 1984.

4. http://www.gisdatadepot.com

5. M. Garey, D. Johnson, F. Preparata and R. Tarjan, "Triangulating a simple polygon", Inform. Proc. Lett., 7, pp. 175-180, 1978.

6. G. Lorenzetto, A. Datta and R. Thomas, "A fast trapezoidation technique for planar polygons", Computers \& Graphics, March 2002, to appear.

7. B. Žalik and G. J. Clapworthy, "A universal trapezoidation algorithm for planar polygons", Computers \& Graphics, 23, pp. 353-363, 1999. 